\section{Twelve-year analysis of cattle and buffalo slaughtering in Lazio Region (2000-2012): animal husbandry and veterinary public health implications}

\author{
Selene Marozzi, ${ }^{1}$ Paola Scaramozzino, \\ Renato Colafrancesco ${ }^{2}$
}

'Direzione Operativa Controllo degli

Alimenti ed Ittiopatologia, Istituto

Zooprofilattico Sperimentale delle

Regioni Lazio e Toscana; ${ }^{2}$ Centro

Operativo per l'Anagrafe Zootecnica e

Sistema Informativo di Epidemiologia

Veterinaria, Istituto Zooprofilattico

Sperimentale delle Regioni Lazio e

Toscana, Roma, Italy

\section{Abstract}

In recent years, beef meat chain has undergone major transformations due to Community legislation and market changes. The purpose of this work is to analyse the information recorded in Banca Dati Nazionale (BDN; Italian computerised database for the identification and registration of bovine animals) on cattle and buffaloes slaughtered between 2000 and 2012 and related to Lazio Region as a result of breeding and/or slaughtering place. The analysis of the data showed a negative trend (-20.7\%) for cattle slaughtered from 2000 to 2012 . Most of this animals had been raised in Lazio Region (86\%) and in particular in the province of Frosinone. The average age at slaughter for female is about 4 years (1417 days) and for males of 547 days. The buffaloes, however, are intended for slaughter at an average age of about 8 years, if female, and about one year if male.

\section{Introduzione}

Nel panorama agroalimentare italiano, la filiera delle carni riveste un ruolo di primaria importanza rappresentando poco meno di un quarto del valore complessivo dell'industria agroalimentare (ISMEA, 2003). Il settore della carne bovina, in particolare, è una delle componenti principali del comparto e negli ultimi anni ha subito profonde trasformazioni organizzative e strutturali. Queste ultime sono state in parte influenzate dai cambiamenti della normativa comunitaria di riferimento e dai mutamenti degli equilibri di mercato consequenziali alla crisi sanitaria dell'encefalopatia spongiforme bovina (BSE). Nell'UE è stato introdotto un cospicuo corpus di leggi per tutelare l'uomo e gli animali dalla BSE e da altre TSE. Le principali misure adottate hanno riguardato nello specifico il divieto di impiegare proteine animali nei mangimi destinati ad animali allevati per la produzione alimentare ed un insieme di analisi ed accertamenti sugli animali macellati e venuti a morte in allevamento. Inoltre, al fine di migliorare l'efficienza dei controlli (rintracciabilità) e la trasparenza in merito alle condizioni di produzione e commercializzazione delle carni è stato istituito, da un lato, un sistema efficace di identificazione e di registrazione dei bovini e, dall'altro, sono state disciplinate le informazioni inerenti l'etichettatura delle carni bovine. Per raggiungere tali obiettivi l'Unione Europea ha promulgato il Regolamento (CE) 1760/2000 (Commissione Europea, 2000) che ha istituito un sistema di identificazione e di registrazione dei bovini, ha predisposto la creazione di una banca dati informatizzata contenente i dati delle aziende, degli allevamenti e dei singoli animali presenti sul territorio di competenza ed ha definito le modalità obbligatorie di etichettatura delle carni e dei prodotti a base di carni bovine. Successivamente l'Italia, con il Decreto del Ministero della Salute del 31 Gennaio 2002 (Ministero della Salute, 2002), Disposizione in materia di funzionamento dell'anagrafe bovina, ha designato la propria Banca Dati Nazionale (BDN). Secondo le leggi cogenti gli obblighi informativi sui capi bovini devono essere valorizzati direttamente dal detentore dell'animale 0 suo delegato (nascita dell'animale, morte, movimentazioni in entrata e uscita dall'allevamento) e dai titolari degli stabilimenti di macellazione per quanto attiene le informazioni sui capi macellati. L'Articolo 8 , in particolare, demanda al responsabile dello stabilimento di macellazione preventivamente registrato, la comunicazione per via informatica alla BDN, delle informazioni relative ai capi macellati, e provvede, sotto controllo del servizio veterinario, alla distruzione dei marchi auricolari degli animali macellati.

La Regione Lazio con Delibera della Giunta Regionale 2 Agosto 2002 n. 1096 (Regione Lazio, 2002), si è dotata di una propria Banca Dati Regionale (BDR) aggiornata ed allineata quotidianamente con i dati provenienti dalla BDN. La BDR consente la valorizzazione dei dati anagrafici in ambito locale permettendo di utilizzare gli stessi per interventi di epidemiosorveglianza, sanità pubblica, sicurezza alimentare e benessere animale.

Scopo del presente lavoro è analizzare le informazioni registrate in BDR sui capi bovini e bufalini macellati nel periodo 2000-2012 ed afferenti, per provenienza o sede di macellazione, alla Regione Lazio. Tutte le analisi sono state condotte correlando i dati di cui sopra ad alcune variabili individuali (provenienza capo, luogo macellazione, età, sesso) ed al patrimo-
Correspondence: Renato Colafrancesco, Centro Operativo per l'Anagrafe Zootecnica e Sistema Informativo di Epidemiologia Veterinaria, Istituto Zooprofilattico Sperimentale delle Regioni Lazio e Toscana, via Appia Nuova 1411, 00178 Roma, Italy.

Tel. +39.06.7099325 - Fax: +39.06 .79340724 .

E-mail: renato.colafrancesco@izslt.it

Key words: Cattle slaughtering, Buffalo slaughtering, Veterinary public health.

Received for publication: 10 May 2013.

Accepted for publication: 6 September 2013.

This work is licensed under a Creative Commons Attribution 3.0 License (by-nc 3.0).

(C) Copyright S. Marozzi et al., 2014

Licensee PAGEPress, Italy

Italian Journal of Food Safety 2014; 3:1665

doi:10.4081/ijfs.2014.1665

nio zootecnico regionale e nazionale al fine di evidenziare i trend in campo zootecnico e le eventuali implicazioni sanitarie.

\section{Materiali e Metodi}

Le informazioni sui capi macellati sono state estratte dal database informatico della BDR, aggiornato quotidianamente, mediante tecnologia web services, con i dati contenuti nella BDN. Le interrogazioni informatiche (query) sono state effettuate considerando i seguenti criteri di ricerca ed aggregazione: specie animale (bovini e bufalini), periodo di riferimento (2000-2012), regione di riferimento (Lazio), provenienza territoriale dei capi bovini e bufalini, intesa come azienda di ultima detenzione, luogo di macellazione, età alla macellazione dei capi, sesso. I dati residenti in un database MySql (Oracle Corporation) sono stati analizzati al fine di eliminare i capi con un anagrafica non correttamente registrata ed una provenienza territoriale incerta. Il numero dei capi macellati è stato quindi esaminato in relazione alle variabili anno di macellazione, provenienza, luogo di macellazione, sesso ed età e correlato al patrimonio zootecnico nazionale e regionale acquisibile, quest'ultimo, dal portale del Ministero della Salute (https://www. vetinfo.sanita.it/). A tal fine sono stati considerati i seguenti criteri: specie animale (bovini e bufalini), periodo di riferimento (2004-2012), luogo di riferimento (Italia, Lazio).

\section{Risultati}

Sono stati eliminati dalla elaborazione 
$n^{\circ} 411$ capi per i quali la data di nascita risultava essere maggiore 0 uguale alla data di macellazione, $\mathrm{n}^{\circ} 201$ capi con data di nascita precedente al 1980 ed con codice azienda di provenienza errato. Ai fini dello studio sono stati pertanto considerati 1.005.524 soggetti di cui 956.399 bovini $(95,1 \%)$ e 49.125 bufalini $(4,9 \%)$. Le macellazioni bovine e bufaline regionali rappresentano in media l' $1,9 \%$ del totale macellazioni nazionali (media capi/anno: 59.045) (Tabella 1). Le Regioni Veneto, Lombardia, Piemonte ed Emilia Romagna rappresentano da sole il $77,6 \%$ del volume totale di capi bovini e bufalini macella- ti. Nel corso dei dodici anni si è verificata, in concomitanza con la diminuzione del patrimonio zootecnico (-10\% Italia; -16,7\% Regione Lazio), una significativa variazione percentuale del numero di capi macellati sia a livello nazionale (-15,6\%; range capi: $3.408 .972-$ $2.876 .832)$ che locale $(-20,7 \%$; range capi: 65.990-52.361) (Tabella 1). Più in dettaglio, per quanto attiene la diminuzione del patrimonio zootecnico, l'analisi dei dati per singola specie mostra che il trend negativo è da attribuirsi alla flessione registrata per la specie bovina (-25,1\% Regione Lazio; $-12 \%$ Italia) mentre per i bufalini si è assistito ad un incre- mento dei soggetti allevati $(+28,8 \%$ Regione Lazio; $+32,3 \%$ Italia)

I bovini di provenienza regionale macellati nel Lazio (Tabella 2) si attestano mediamente intorno al $21,4 \%$ del patrimonio zootecnico locale (Tabella 1). Gli stabilimenti di macellazione processano soprattutto capi bovini provenienti dal territorio regionale $(86,8 \%)$ (tabella 2) ed in particolar modo dalle province di Frosinone (28,6\%; $n=181.916)$, Roma $(23,6 \% ; \quad \mathrm{n}=150.278)$ e Latina $(20,5 \%$; $\mathrm{n}=130.436$ ).

In relazione al luogo di allevamento e di macellazione dei capi è interessante rilevare

Tabella 1. Patrimonio zootecnico bovino e bufalino e numero dicapi macellati nella Regione Lazio e in Italia. Fonte dati: Banca Dati Nazionale; utlimo accesso: Aprile 2013.

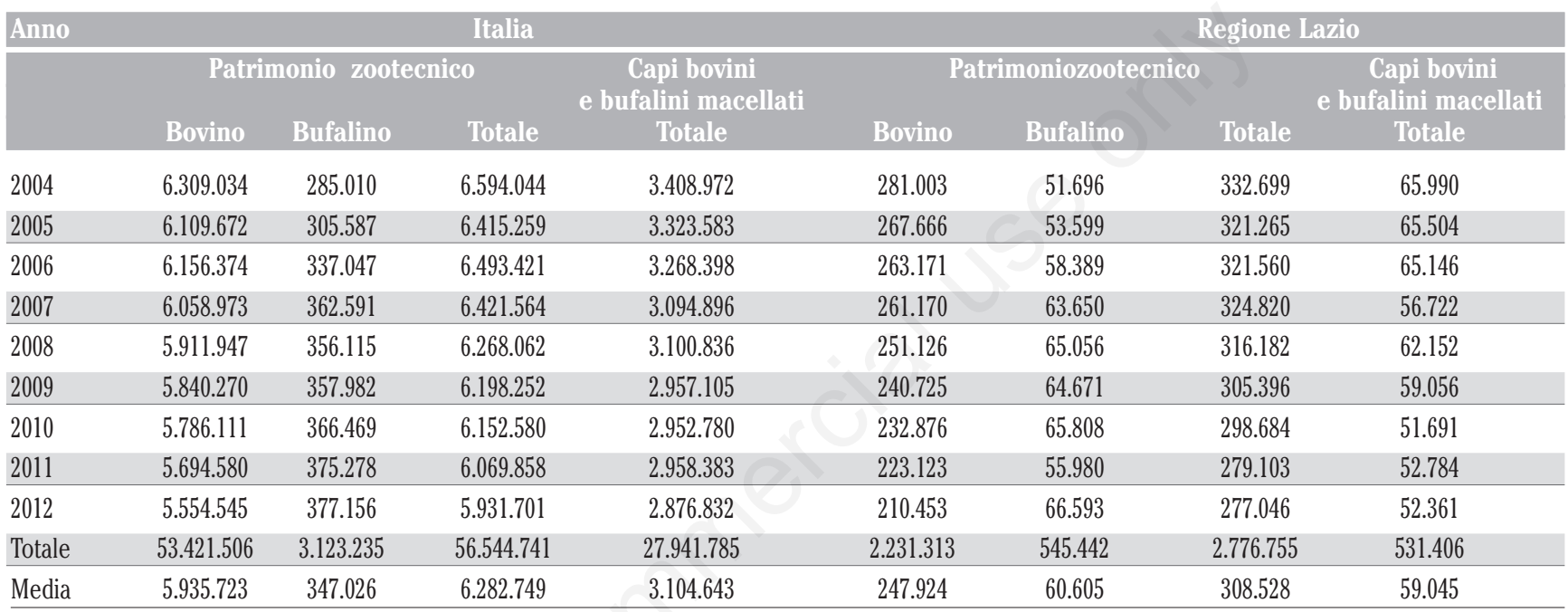

Tabella 2. Bovini e bufalini macellati nella Regione Lazio dal 2000 al 2012. Fonte dati: Banca Dati Nazionale; utlimo accesso: Aprile 2013.

\begin{tabular}{|c|c|c|c|c|c|c|c|c|}
\hline \multirow[t]{2}{*}{ Anno } & \multicolumn{2}{|c|}{$\begin{array}{l}\text { Capi di provenienza } \\
\text { regionale }\end{array}$} & $\begin{array}{r}\text { acellati } \\
\text { Cap } \\
\text { extr }\end{array}$ & $\begin{array}{l}\text { regione I } \\
\text { rovenienza } \\
\text { sionale }\end{array}$ & zio & & \multicolumn{2}{|c|}{$\begin{array}{c}\text { Capi macellati fuori regione Lazio } \\
\text { Capi di provenienza }\end{array}$} \\
\hline & Bovini & Bufalini & Bovini & Bufalini & Bovini & Bufalini & Bovini & Bufalini \\
\hline 2000 & 31680 & 22 & 3657 & 9 & 35337 & 31 & 10470 & 57 \\
\hline 2001 & 43089 & 65 & 4263 & 33 & 47352 & 98 & 8867 & 79 \\
\hline 2002 & 47545 & 82 & 5343 & 48 & 52888 & 130 & 16891 & 266 \\
\hline 2003 & 50779 & 214 & 5687 & 24 & 56466 & 238 & 20193 & 328 \\
\hline 2004 & 60100 & 212 & 7434 & 1 & 67534 & 213 & 22543 & 434 \\
\hline 2005 & 62097 & 949 & 9365 & 22 & 71462 & 971 & 21053 & 1353 \\
\hline 2006 & 57049 & 2062 & 10206 & 14 & 67255 & 2076 & 18730 & 2211 \\
\hline 2007 & 53739 & 2498 & 9631 & 21 & 63370 & 2519 & 17296 & 2674 \\
\hline 2008 & 54459 & 4241 & 10101 & 68 & 64560 & 4309 & 19896 & 2799 \\
\hline 2009 & 47184 & 3296 & 8934 & 18 & 56118 & 3314 & 16802 & 3506 \\
\hline 2010 & 44743 & 2826 & 7664 & 48 & 52407 & 2874 & 16545 & 3182 \\
\hline 2011 & 47002 & 3397 & 9877 & 87 & 56879 & 3484 & 17621 & 4007 \\
\hline 2012 & 37079 & 3467 & 5007 & 374 & 42086 & 3841 & 15778 & 4131 \\
\hline Totale & 636545 & 23331 & 97169 & 767 & 733714 & 24098 & 222685 & 25027 \\
\hline Media & 53045,4 & 1944,3 & 8097,4 & 63,9 & 61142,8 & 2008,2 & 18557,1 & 2085,6 \\
\hline
\end{tabular}


che la maggior parte della produzione (Frosinone: 94,1\%; Latina: 70,5\%; Rieti: 94,1\%; Viterbo: 94,9\%) viene destinata a macelli della stessa provincia di ingrasso, ciò ad esclusione di Roma (33\%), che invia i propri capi anche a stabilimenti delle province di Viterbo $(38,6 \%)$ e Frosinone (34,6\%). Per quanto riguarda i bufalini, analogo discorso vale per Rieti (98,9\%), Frosinone $(96,1 \%)$ e Viterbo $(94,4 \%)$. Le province di Latina e Roma, invece, inviano circa la metà della loro produzione $(51,5 \%$; $51,7 \%)$ agli stabilimenti di macellazione di Frosinone. I bovini allevati sul territorio regionale e macellati in stabilimenti di altre Regioni rappresentano il $25,9 \%$ del totale bovini laziali macellati (tabella 2) e provengono in massima parte dalla provincia di Roma (29,5\%; $\mathrm{n}=65.671$ ). Il 43,7\% dei soggetti abbattuti in altre Regioni viene inviato in Lombardia (media capi/anno: 8.111), il $16,6 \%$ in Campania (media capi/anno: 3.007 ) ed il 14,1\% in Emilia Romagna (media capi/anno: 2.627). Per quanto concerne i bufalini, invece, è la provincia di Latina ad essere la zona di provenienza della maggior parte dei capi regionali inviati nei mattatoi laziali $(67,8 \%$; $\mathrm{n}=15.815)$, 0 in altri stabilimenti extra regionali $(62,8 \% ; n=15.706)$.

La Regione dalla quale proviene il maggior numero di capi bovini extra regionali macellati negli stabilimenti del Lazio è il Veneto $(30,6 \%)$, seguito dall'Umbria $(13,3 \%)$ e dalla Toscana (13,1\%). Per quanto concerne i bufalini, è la Campania $(80,2 \%)$ a sopperire da sola alla quasi totalità delle macellazioni di capi in arrivo da altre Regioni.

Il sesso dei bovini allevati e macellati nella Regione risulta essere per il $41,3 \%$ femminile e per il $58,7 \%$ maschile. I soggetti di sesso femminile provenienti dal Lazio vengono abbattuti ad un età media di circa 4 anni (1417 giorni) mentre quelli di sesso maschile a circa un anno e mezzo dalla nascita (547 giorni).

Per quanto concerne i bovini di provenienza extra regionale questi sono in prevalenza di sesso maschile $(66,7 \%)$ ed hanno un'età media di circa 1,3 anni (478 giorni). I capi di sesso femminile allevati nel Lazio e macellati nel territorio regionale hanno mediamente un'età minore (45,6 mesi) rispetto a quelli abbattuti in stabilimenti extra regionali $(82,9$ mesi). Considerando in totale i capi bovini di provenienza regionale ed extraregionale le femmine sono il $40,2 \%$ del totale e vengono abbattute ad un'età media di 4 anni $(48,4$ mesi) mentre i maschi rappresentano il 59,8\% e vengono inviati alla macellazione dopo 504 giorni (16,8 mesi) dalla nascita. Nel corso degli anni si è verificato un incremento dell'età alla macellazione dei capi bovini di sesso femminile allevati nel Lazio o provenienti da altre Regioni (range 2000-2012: 50,3- 61,6 mesi). Al contrario per quelli di sesso maschile il dato è sostanzialmente invariato (range 2000-2012: 18,6-17,7 mesi).

Relativamente ai capi bufalini allevati e macellati nella Regione, i soggetti di sesso femminile risultano essere il $38,2 \%$ e vengono inviati agli stabilimenti regionali per la macellazione ad un età media di circa 8 anni (3.069 giorni) mentre quelli di sesso maschile $(61,8 \%)$ a circa un anno dalla nascita (432 giorni) (Tabella 3).

\section{Discussione Conclusioni}

Le recenti crisi sanitarie scaturite dall'identificazione di carne di specie equina in prodotti dichiarati a base di carne bovina hanno ribadito la valenza sanitaria della corretta identificazione anagrafica del bestiame per finalità di epidemiosorveglianza e di sicurezza alimentare. L'anagrafe zootecnica bovina, in particolare può rappresentare uno strumento elettivo per studi epidemiologici in sicurezza alimentare. Per quanto attiene la Regione Lazio, l'analisi dodecennale delle macellazioni mostra alcuni aspetti rilevanti in termini zootecnici ed epidemiologici. Relativamente agli stabilimenti di macellazione che lavorano capi delle specie bovina e bufalina, il Lazio vanta attualmente 33 attività censite nel database ministeriale (Ministero della Salute, 2013) distribuite in maniera non omogenea sul territorio. In parti- colare sono le province di Frosinone, Rieti e Viterbo rispettivamente con $\mathrm{n} 10, \mathrm{n} 9$ ed n 8 stabilimenti quelle nelle quali si concentra il maggior numero di impianti. I dati estratti dalla BDR hanno evidenziato che i maggiori volumi di macellazione per i bovini si concentrano nelle province di Frosinone e Viterbo, mentre, la provincia di Rieti, sebbene sia dotata di 9 stabilimenti di macellazione, copre una quota inferiore rispetto alle prime due. Ciò è dovuto probabilmente all'inferiore capacità lavorativa degli impianti reatini rispetto ai mattatoi del frusinate e della tuscia.

Come avvalorato dai dati sui capi allevati nel Lazio e macellati negli stabilimenti regionali ( $86 \%$ del totale) e da quelli relativi alla provincia di provenienza e di abbattimento, in riferimento alle prime fasi della catena produttiva, la filiera della carne bovina regionale si configura come breve e strettamente localizzata nel territorio di origine degli animali. Ciò ad esclusione della sola provincia di Roma dove i 3 stabilimenti di macellazioni presenti risultano insufficienti a sopperire al carico produttivo con una consequenziale decentralizzazione delle attività verso altre province del Lazio e verso altre Regioni. Altro dato rilevante riguarda invece le caratteristiche di sesso ed età. Infatti i bovini di sesso femminile ed età di circa 4 anni rappresentano il 40,2\% del totale macellati nei 12 anni presi in esame. Tale valore comprende quindi una quota rilevante di capi a fine carriera provenienti da indirizzi zootecnici specifici per la produzione di latte 0 di riproduttori destinati alla macellazione. La categoria commerciale più rappresentata per capi bovini di sesso maschile, sembrerebbe essere invece il vitellone (15/18 mesi peso 500 $600 \mathrm{~kg}$ ). Da un punto di vista agroozotecnico è possibile distinguere la produzione di carne bovina in tre segmenti commerciali: il vitello a carne bianca, la vacca di fine carriera, il vitellone maschio o femmina (scottona). Il peso in termini economici a livello nazionale di quest'ultimo segmento, è pari al 74\% dell'offerta complessiva (ISMEA, 2006). L'analisi dei dati sulle macellazioni nella Regione Lazio evidenzia una tendenza certamente in linea con l'am-

Tabella 3. Bovini e bufalini di provenienza regionale macellati nel Lazio in relazione al sesso e all'età (2000-2012). Fonte dati: Banca Dati Nazionale; utlimo accesso: Aprile 2013.

\begin{tabular}{|c|c|c|c|c|c|c|}
\hline Specie & Sesso & n & Età media in giorni & STD & Min & Max \\
\hline Bovini & $\mathrm{F}$ & 262641 & 1417 & 1376 & 1 & 10670 \\
\hline Bovini & M & 373877 & 547 & 277 & 1 & 8043 \\
\hline Totale & & 636518 & 906 & 1005 & 1 & 10670 \\
\hline Bufalini & $\mathrm{F}$ & 8906 & 3069 & 1930 & 2 & 11301 \\
\hline Bufalini & M & 14425 & 432 & 525 & 1 & 7107 \\
\hline Totale & & 23331 & 1439 & 1798 & 1 & 11301 \\
\hline
\end{tabular}

STD, solidi totali disciolti. 
bito di pertinenza nazionale ma comunque indirizzata anche verso il segmento commerciale vacche a fine carriera. Ciò probabilmente è determinato dalla vocazione del comparto zootecnico regionale maggiormente incentrato verso la produzione di latte. Infine così come avvalorato dall'età media dei soggetti femminili che risulta essere notevolmente più alta per le vacche a fine carriera allevate nel Lazio ma abbattute in altre Regioni, è possibile ipotizzare che le lattifere al termine della vita produttiva, vengano destinate all'abbattimento soprattutto in stabilimenti extra regionali. Questa condizione troverebbe giustificazione nella localizzazione, in territorio extra regionale, di un numero maggiore di impianti di trasformazione delle carni, attività indirizzate all'uso per fini tecnologici ed economici, di materie prime meno pregiate.

\section{Bibliografia}

Commissione Europea, 2000. Regolamento del Parlamento Europeo e del Consiglio del 17 luglio 2000 che istituisce un sistema di identificazione e di registrazione dei bovini e relativo all'etichettatura delle carni bovine e dei prodotti a base di carni bovine, e che abroga il Regolamento (CE) n. 820/97 del Consiglio Europeo, 1760/2000/CE. In: Gazzetta ufficiale, L 204/1.

ISMEA, 2003. Filiera animale e carni: ricerche. Quaderno di filiera n. 16 - statistiche del settore carne. Disponbile al sito: http://www.assocarni.it/pagina13_dossier.html

ISMEA, 2006. Analisi del costo e della redditivi- tà della produzione di carne bovina in Italia- Indagine 2006. Redazione a cura della direzione mercati e risk management. Istituto di Servizi per il Mercato Agricolo Alimentare ed., Roma, Italia.

Ministero della Salute, 2002. Decreto 31 gennaio 2002. Disposizione in materia di funzionamento dell'anagrafe bovina. In: Gazzetta ufficiale, L 72, 26/03/2002.

Ministero della Salute, 2013. Disponbile al sito: http://alimvet.sanita.it/NuovoStabilimenti/

Regione Lazio, 2002. Deliberazione della giunta Regionale 2 Agosto 2002, n 1096 avente per oggetto: decreto ministeriale 31 Gennaio 2002, art. 1, comma 3 . Definizione delle fasi gestionali dell'anagrafe bovina regionale. Regione Lazio ed., Roma, Italia. 